\title{
Solving the population balance equation for granulation processes: particle layering and agglomeration
}

\author{
Ludmila Vesjolaja $^{1} \quad$ Bjørn Glemmestad ${ }^{2} \quad$ Bernt Lie $^{1}$ \\ ${ }^{1}$ Department of Electrical Engineering, IT and Cybernetics, University of South-Eastern Norway, \\ \{ludmila.vesjolaja, bernt.lie\}@usn.no \\ ${ }^{2}$ Process Modeling and Control Department, Yara Technology Center, Norway, bjorn.glemmestadeyara.com
}

\begin{abstract}
Granulation processes are frequently used in the fertilizer industry to produce different grades of mineral fertilizers. Large recycle ratios and poor product quality control are some of the problems faced by such industries. Thus, for real time model based process control and optimization, it is necessary to find an appropriate numerical scheme can find solution of the model sufficiently accurate and fast. In this study, population balance principles were used to model particle granulation processes. Different numerical schemes were tested to find simple yet sufficiently accurate solution schemes for population balance equation. Numerical schemes were applied to find the solution of both the layering term and the agglomeration term that appear in the population balance equation. The accuracies of the numerical schemes were assessed by comparing the numerical results with analytical, tractable solutions. Comparison of the accuracy of numerical schemes showed that a high resolution scheme with Koren flux limiter function might be a good choice for the layering term discretization, while a cell averaging technique and a new finite volume method of Kumar et al. (2016) produce a sufficiently accurate solution for the agglomer-ation term discretization.
\end{abstract}

Keywords: population balance, numerical scheme, layering, agglomeration

\section{Introduction}

Granulation is a particle enlargement process during which fine particles and/or atomizable liquids are converted into granules via a series of complex physical processes (Litster and Ennis, 2004). Here, the focus is on modeling a granulation process used for mineral fertilizer production. In the fertilizer industry, depending on desired product properties, different types of granulators are used, e.g., spherodizers and drum granulators.

Formation of the particles (granulation mechanisms) depends on the granulator type and operating conditions. Particle growth due to layering is predominant in spherodizers. Layering is a continuous process (differential growth) during which particle growth occurs due to a successive coating of a liquid phase onto a granule (Litster and Ennis, 2004). In drum granulators, on the other

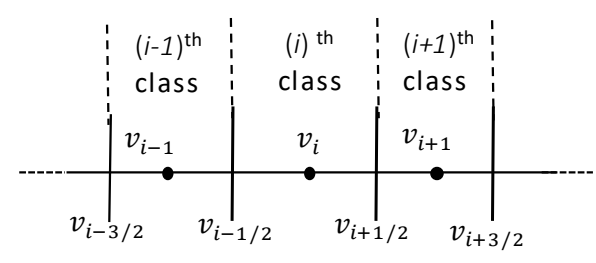

Figure 1. Size discretization into classes (cells) using linear grid.

hand, particle collision occurs, and thus particle agglomeration contributes significantly to particle size change. In this paper, binary particle agglomeration is assumed for population balance $(\mathrm{PB})$ modeling. Binary agglomeration refers to a particle growth mechanism that occurs due to successful collision of two particles, resulting in the formation of a larger, composite particle. Thus, the agglomeration results in a reduction of the total number of particles, while the total mass remains conserved (Litster and Ennis, 2004).

The operation of granulation plants at an industrial scale can be challenging (Litster and Ennis, 2004; Radichkov et al., 2006; Heinrich et al., 2003). Periodic instability associated with the operation of the granulation circuit has been reported. This causes the particle size distribution (PSD) flowing out of the granulator to oscillate, thus making it difficult to maintain the desired product quality, e.g., particle size. Thus, to address and solve these challenges, it is essential to have a dynamic model of the granulator that can further be used to design optimal control structures. The model should be both simple and sufficiently accurate to reflect the underlying physical mechanisms that take place in the granulator. The resulting population balance equations (PBEs) are nonlinear in nature and are challenging to solve. Analytical solutions of these PBEs are available only for ideal and simplified cases, and thus for most of the cases, numerical methods are needed to solve such PBEs. For real time model based process control and optimization, it is necessary to find an appropriate numerical scheme that is sufficiently accurate and fast. Therefore, the main focus 
of this paper has been (i) investigation of the accuracy of different numerical schemes that are suitable for discretizing PBEs by comparing the numerical results with analytically tractable solutions; (ii) application of various finite volume techniques (first order upwind, second order central difference, and a high resolution scheme) to the growth by a layering process; (iii) application of different sectional methods (Hounslow method, cell average technique, fixed pivot scheme), and a new finite volume method of Kumar et al. (2016) to the agglomeration process.

\section{Population Balance Equation (PBE)}

Population balance (PB) is frequently used to describe dynamics of particle property distributions, e.g., particle size distribution, moisture content in particles and porosity (Ramkrishna, 2000). The general form of a number based PBE with particle size $(x)$ as the internal coordinate, spatial variation $(z)$ as the external coordinate, and time $t$ as time coordinate is represented as

$$
\begin{aligned}
\frac{\partial n(x, z, t)}{\partial t}=-\frac{\partial}{\partial x}[G n(x, z, t)] & +B(x, z, t)-D(x, z, t) \\
& -\frac{\partial}{\partial z}\left[\frac{d z}{d t} n(x, z, t)\right],
\end{aligned}
$$

where $n(x, z, t)$ is the number density function $\left[\frac{n o}{\mathrm{~mm}^{3} \cdot[\text { internal coordinate }]}\right]$. The first term on the right hand side represents the particle growth due to layering, the second and third terms stand for particle birth and death, respectively, due to agglomeration, while the last term represents a continuous process and gives the flow of particles through the granulator. $G$ is the growth rate $\left[\frac{\text { internal coordinate }}{s}\right]$ (Ramkrishna, 2000). The birth $B$ and death $D$ terms usually include integrals that lead to partial integro-differential equations which make the solution of the PBEs complicated. Mathematical expressions for birth and death terms are shown in Section 4 when describing different numerical schemes for binary agglomeration. Further simplifications of the general PBE (Eq. 1) are possible and are dependent on the nature of the process taken into consideration.

At this point, it is convenient to define moments of particle size distributions that will be used later for discussion of the simulation results. The $l$-th moment of the PSD is defined as

$$
\mu^{l}=\int_{0}^{\infty} x^{l} n(x) d x
$$

The first moments are of particular interest. Depending on the choice of internal coordinate (e.g., particle volume or particle length) the moments are related to the total number, length, area, and volume of particles.

\section{Numerical schemes for layering term discretization}

PBEs are non-linear in general and analytical solutions are available only for simplified processes. Thus, a simple 1-D batch process for which an analytical solution is available is modeled so that the accuracy of the numerical schemes can be reliably evaluated. In a batch granulation process there is no continuous particle flow through the granulator. If the particle size change in the granulator is mainly due to layering (e.g., in spherodizers), and the size of a particle is represented by its volume $v$, then Eq. 1 reduces to

$$
\frac{\partial n(v, t)}{\partial t}=-\frac{\partial}{\partial v}[G n(v, t)] .
$$

In Eq. 3, the concept of perfect mixing inside the granulator is applied: particle property (e.g., size distribution) inside the granulator is the same at every point inside the granulator. In this paper, the solution to PBEs containing growth term $G$ (Eq. 3) is found by transforming the partial differential equation (PDE) into a system of ordinary differential equations (ODEs), i.e., by reducing the dimensionality of the problem with respect to the particle size. The set of ODEs can then be solved using an appropriate time integrator. In this paper, a Runge-Kutta 4-th order (RK-4) time integration method is used for all simulations.

For particle size discretization, first the particles are classified into $N_{c}$ particle classes which are numbered by $i \in\left\{1,2, \ldots N_{c}\right\}$ classes (cells) using a linear grid as shown in Figure 1.

Here, $i$ represents the $i$-th particle class, $v_{i}$ is the volume of the particle of the $i$-th class, $v_{i \pm \frac{1}{2}}$ is the left and the right boundaries of the $i$-th class, and $\triangle v=v_{i+\frac{1}{2}}-v_{i-\frac{1}{2}}$ is the size of the classes. The dots in each class (Figure 1) represent the cell centers. Secondly, an appropriate numerical scheme is applied to convert Eq. 3 into set of ODEs. Integration of Eq. 3 over cell $i$ from $v_{i-\frac{1}{2}}$ to $v_{i+\frac{1}{2}}$ gives

$$
\frac{d N_{i}(t)}{d t}=G\left(v_{i-\frac{1}{2}}\right) n\left(v_{i-\frac{1}{2}}, t\right)-G\left(v_{i+\frac{1}{2}}\right) n\left(v_{i+\frac{1}{2}}, t\right) .
$$

In a simplified case, a solution of Eq. 4 can be found analytically: The particle growth due to layering in a batch process does not change the total number of particles in the batch, but only the particle volume is changed. If $t$ denotes the time for particle growth, $v_{\text {initial }}$ denotes the initial volume of the particles (for all classes), and $G$ is the constant growth rate, then the new volume $\left(v_{\text {new }}\right)$ of the particles after the growth due to layering is given as

$$
v_{\text {new }}=v_{\text {initial }}+t \cdot G \text {. }
$$

However, in real applications, analytical solutions are difficult to obtain, and, thus various numerical schemes are applied to approximate the right hand side of Eq. 4. The 
PBE represented by Eq. 3 is a hyperbolic equation due to the layering term, and Eq. 3 can be approximated using a finite volume scheme that automatically incorporates conservation of number in a growth process.

In this paper, three finite volume schemes are compared for particle size discretization, namely a first order upwind scheme (FU) and a second order central difference scheme (SCD). The first order upwind (FU) scheme uses the approximation defined by Eq. 6 and 7:

$$
n\left(v_{i-\frac{1}{2}}, t\right) \approx \frac{1}{\Delta v}\left[N_{i-1}(t)\right]
$$

and

$$
n\left(v_{i+\frac{1}{2}}, t\right) \approx \frac{1}{\Delta v}\left[N_{i}(t)\right]
$$

Thus, approximation of Eq. 4 using the FU scheme leads to

$$
\frac{d N_{i}}{d t} \approx \frac{1}{\Delta v}\left[G\left(v_{i-\frac{1}{2}}\right) N_{i-1}(t)-G\left(v_{i+\frac{1}{2}}\right) N_{i}(t)\right] .
$$

Assuming constant growth rate in all cells, Eq. 8 simplifies to

$$
\frac{d N_{i}^{\mathrm{FU}}}{d t} \approx \frac{G}{\Delta v}\left[N_{i-1}(t)-N_{i}(t)\right]
$$

The second order central difference (SCD) scheme uses the approximation

$$
n\left(v_{i-\frac{1}{2}}, t\right) \approx \frac{1}{\Delta v} \frac{\left[N_{i-1}(t)+N_{i}(t)\right]}{2}
$$

and

$$
n\left(v_{i+\frac{1}{2}}, t\right) \approx \frac{1}{\Delta v} \frac{\left[N_{i}(t)+N_{i+1}(t)\right]}{2} .
$$

Thus, discretization of Eq. 4 over a cell $i$ using the SCD scheme results in

$$
\frac{d N_{i}^{\mathrm{SCD}}}{d t} \approx \frac{G}{\Delta v} \frac{\left[N_{i-1}(t)-N_{i+1}(t)\right]}{2}
$$

where particle growth $G$ is assumed constant in all the cells.

A finite volume scheme that is extended by a flux limiter is also applied to Eq. 4 to reduce the dimensionality of the PBEs with respect to the particle size. In particular, the Koren flux limiter function (Koren, 1993) is used to achieve a robust upwind discretization scheme to Eq. 4. High resolution schemes are considered to attain higher accuracy than the first order upwind schemes. In addition, these methods avoid spurious oscillations by applying a high order flux in the smooth regions and a low order flux near discontinuities (Koren, 1993; Kumar, 2006). Assuming constant $G$ in all cells, Eq. 4 can be discretized with the Koren scheme as

$$
\frac{d N_{i}^{\mathrm{KFL}}}{d t} \approx G \cdot\left[n\left(v_{i-\frac{1}{2}}\right)-n\left(v_{i+\frac{1}{2}}\right)\right]
$$

where,

$$
\begin{aligned}
n\left(v_{i-\frac{1}{2}}, t\right) \approx & \frac{1}{\Delta v}\left[N_{i-1}(t)\right. \\
& \left.\times \frac{1}{2} \phi\left(\widetilde{\theta}_{i-\frac{1}{2}}\right) \cdot\left(N_{i-1}(t)-N_{i-2}(t)\right)\right],
\end{aligned}
$$

and

$n\left(v_{i+\frac{1}{2}}, t\right) \approx \frac{1}{\Delta v}\left[N_{i}(t)+\frac{1}{2} \phi\left(\widetilde{\theta}_{i+\frac{1}{2}}\right) \cdot\left(N_{i}(t)-N_{i-1}(t)\right)\right]$.

Here, $\phi$ is the limiter function defined as

$$
\phi(\widetilde{\theta})=\max \left[0, \min \left(2 \widetilde{\theta}, \min \left(\frac{1}{3}+\frac{2 \widetilde{\theta}}{3}, 2\right)\right)\right] .
$$

Parameter $\widetilde{\theta}$ is defined as,

$$
\widetilde{\theta}_{i-\frac{1}{2}}=\frac{N_{i}-N_{i-1}+\chi}{N_{i-1}-N_{i-2}+\chi}, \quad \widetilde{\theta}_{i+\frac{1}{2}}=\frac{N_{i+1}-N_{i}+\chi}{N_{i}-N_{i-1}+\chi},
$$

with a very small constant $\chi$ (e.g., $10^{-8}$ ) to avoid division by zero.

\section{Numerical schemes for agglomera- tion term discretization}

PBE for a batch agglomeration process using the particle volume as internal coordinate is given by

$$
\frac{\partial n(v, z, t)}{\partial t}=B(v, z, t)-D(v, z, t)
$$

Here, the particle birth $(B)$ and death $(D)$ due to binary agglomeration are modeled using the Hulburt and Katz formulation (Hulburt and Katz, 1964). For a pure agglomeration process, the Hulburt and Katz equation (Hulburt and Katz, 1964) is given as

$$
\begin{aligned}
& \frac{\partial n(t, v)}{\partial t}=B-D= \\
& \frac{1}{2} \int_{0}^{v} \beta(t, v-\varepsilon, \varepsilon) n(t, v-\varepsilon) n(t, \varepsilon) d \varepsilon \\
& \quad-n(t, v) \int_{0}^{\infty} \beta(t, v, \varepsilon) n(t, \varepsilon) d \varepsilon .
\end{aligned}
$$

Equation 19 represents a 1-D batch process assuming perfect mixing inside the granulator. In Eq. $19, \beta$ is the agglomeration rate (kernel) that defines the collision frequency of the two particles with volumes $v$ and $v-\varepsilon$.

Agglomeration is a discrete event, and PB modeling of the agglomeration process results in partial integrodifferential equations. The integral function appears in the birth $(B)$ and death $(D)$ terms in Eq. 19. Such systems are difficult to solve, and analytical solutions are available only for a limited number of simplified problems. Some of the analytical solutions for different initial conditions and different agglomeration kernels (e.g., constant, 
sum and product kernels) are given in Scott (Scott, 1968). Here, for simplicity, the performance of different numerical schemes has been assessed using a constant agglomeration kernel with an exponential initial distribution:

$$
n(v, 0)=\frac{N_{0}}{v_{0}} \exp \left(\frac{-v}{v_{0}}\right),
$$

is given as

$$
n(v, t)=\frac{4 N_{0}}{v_{0}(\varpi+2)^{2}} \exp \left(\frac{-2 \kappa}{(\varpi+2)}\right),
$$

In Equations 20 and 21, $N_{0}$ and $v_{0}$ represent the initial number of particles per unit volume and initial mean volume of the particles. The dimensionless volume unit $\kappa$, and the dimensionless time variable $\Phi$ are given by Eq. 22:

$$
\kappa=\frac{v}{v_{0}}, \text { and } \varpi=N_{0} \beta_{0} t
$$

As a result of particle agglomeration, the total number of particles reduces while the total mass remains constant. The main challenge is to find/develop approximation techniques that would assign the newborn particles accurately while conserving the chosen moments. To achieve this, various numerical methods for expressing the agglomeration term in PBEs are developed, among others the method of moments, the method of successive approximations, the finite volume methods, and the sectional methods. A review of various numerical techniques is summarized in (Ramkrishna, 2000). This paper focuses on applying different sectional methods, as well as a newly developed finite volume technique by Kumar et al.'s (Kaur et al., 2017; Singh et al., 2015; Kumar et al., 2016).

Approximation of the continuous size distribution by a finite number of size sections (cells) has been made using a geometric type grids, i.e., the whole particle size interval is divided into a finite number of cells (classes) using geometric progression. This type of grid has been chosen because Hounslow's discretization method, one of the numerical schemes being compared, can be applied only to geometric type grids. The choice of numerical schemes has been made based on suitability for further application of the model for control purposes, i.e., the scheme should posses simplicity in implementation and be sufficiently fast (low computation time), while producing relatively accurate numerical results.

\subsection{Hounslow's scheme}

According to Hounslow's discretization scheme (Hounslow et al., 1988), the approximation of the continuous size distribution by a finite number of cells is performed using a geometric grid with a factor of two in size, i.e., $v_{i+1}=2 v_{i}$. Hounslow's discretization scheme $(\mathrm{H})$ is based on four binary interaction mechanisms that can contribute to changes of particles number in the $i$-th cell. Two of these four mechanisms change the number of particles due to particle births in the $i$-th cell, while the other two mechanisms contribute for particle deaths in the $i$-th cell. Application of the $\mathrm{H}$ scheme to the agglomeration process (Eq. 19) gives the total rate of change of particles in each $i$-th cell as

$$
\begin{array}{r}
\frac{d N_{i}^{\mathrm{H}}}{d t}=\sum_{j=1}^{i-2} 2^{j-i+1} \beta_{i-1, j} N_{i-1} N_{j}+\frac{1}{2} \beta_{i-1, i-1} N_{i-1}^{2}- \\
N_{i} \sum_{j=1}^{i-1} 2^{j-i} \beta_{i, j} N_{j}-N_{i} \sum_{j=i}^{N_{c}} \beta_{i, j} N_{j} .
\end{array}
$$

Here, the first term on the right hand side represents the births of particles that are formed due to collision of particles in the $(i-1)$-th cell with the particles from the first to the $(i-2)$-th cells. The second term on the right hand side stands for the births of particles that are born in cell $i$ by the collision between two particles in the $(i-1)$-th cell. The last two terms in Eq. 23 accounts for the death of particles in the $i$-th cell.

\subsection{Cell average scheme}

The cell average (CA) scheme was introduced by Kumar (Kumar et al., 2006; Kumar, 2006). The CA scheme can be applied to both a geometric grid and a linear grid. Here, a geometric grid discretization has been chosen to be able to compare simulation results with the Hounslow's scheme. In the CA scheme, at first the total birth of particles in each cell denoted by $B_{i}$ is computed:

$$
B_{i}=\sum_{j=1}^{N_{c, i}} B_{i}^{j}=\sum_{j, k}^{j \geqslant k}\left(1-\frac{1}{2} \delta_{j k}\right) \beta_{j k} N_{j} N_{k},
$$

where the two aggregating particles with volumes $v_{j}$ and $v_{k}$ should fulfill the condition $v_{i-\frac{1}{2}} \leq v_{j}+v_{k} \leq v_{i+\frac{1}{2}} ; \delta_{j k}$ is the delta Dirac function such that $\delta_{j k}=1$ for $j=k$, otherwise $\delta_{j k}=0 ; \beta_{j k}$ is the agglomeration kernel for binary agglomeration of particles from the $j$-th and the $k$-th cells.

Then the average volume of the newly formed particles in each cell denoted by $\bar{v}_{i}$ is calculated. The average volume of the particle is then given as

$$
\bar{v}_{i}=\frac{\sum_{j, 1}^{N_{c, i}} y_{i}^{j} B_{i}^{j}}{B_{i}}=\left[\frac{\sum_{j, k}^{j \geqslant k}\left(1-\frac{1}{2} \delta_{j k}\right) \beta_{j k} N_{j} N_{k}\left(v_{j}+v_{k}\right)}{\sum_{j, k}^{j \geqslant k}\left(1-\frac{1}{2} \delta_{j k}\right) \beta_{j k} N_{j} N_{k}}\right] .
$$

The next step in the CA scheme is to assign the total birth of particles $B_{i}$ appropriately to different cells depending on the position of the average volume of all newborn particles relative to the cell center volume $v_{i}$. In total, there are four birth contributions at node $v_{i}$ : two coming from the $i$-th cell itself (when $\bar{v}_{i}<v_{i}$ and $\bar{v}_{i}>v_{i}$ ), and two from the neighboring $(i-1)$-th and $(i+1)$-th cells (when $\bar{v}_{i-1}>v_{i-1}$ and $\left.\bar{v}_{i+1}<v_{i+1}\right)$. To combine all the possible birth contributions, for convenience the dimensionless term $\lambda_{i}^{ \pm}(v)$ is introduced:

$$
\lambda_{i}^{ \pm}(v)=\frac{v-v_{i \pm 1}}{v_{i}-v_{i \pm 1}} .
$$


The discretized PBE using the CA technique takes the form of

$$
\frac{d N_{i}}{d t}=B_{i}^{\mathrm{CA}}-D_{i}^{\mathrm{CA}},
$$

where

$$
\begin{aligned}
B_{i}^{\mathrm{CA}}=B_{i-1} \lambda_{i}^{-}\left(\bar{v}_{i-1}\right) H\left(\bar{v}_{i-1}-v_{i-1}\right) \\
+B_{i} \lambda_{i}^{-}\left(\bar{v}_{i}\right) H\left(v_{i}-\bar{v}_{i}\right)+B_{i} \lambda_{i}^{+}\left(\bar{v}_{i}\right) H\left(\bar{v}_{i}-v_{i}\right) \\
+B_{i+1} \lambda_{i}^{+}\left(\bar{v}_{i+1}\right) H\left(v_{i+1}-\bar{v}_{i+1}\right),
\end{aligned}
$$

and

$$
D_{i}^{\mathrm{CA}}=N_{i} \sum_{k=1}^{N_{c}} \beta_{i, k} N_{k}
$$

Here, the Heaviside step function $H$ is given as

$$
H(v)= \begin{cases}1, & \text { if } v>0 \\ \frac{1}{2}, & \text { if } v=0 \\ 0, & \text { if } v<0\end{cases}
$$

\subsection{Fixed pivot scheme}

The fixed pivot (FP) technique was developed by Kumar and Ramkrishna (Kumar and Ramkrishna, 1996). The scheme is based on the Hounslow's method, however, the disadvantage of using only geometric grids with Hounslow's method is eliminated in the fixed pivot scheme. This scheme can be used with any type of grid including linear grids. Here, for comparison of numerical solutions, the same geometric grid as in the Hounslow and CA schemes has been chosen. The main difference between the FP and CA schemes is in assigning the new-born particles to the cells. In the FP scheme, each individual birth in a cell is directly assigned to the appropriate cells, unlike in the CA scheme where the average volume of all the newly born particles is first calculated, and then only the assignment of the particles is performed.

The discrete form of the PBE with the FP scheme is then written as (Kumar and Ramkrishna, 1996)

$$
\frac{d N_{i}}{d t}=B_{i}^{\mathrm{FP}}-D_{i}^{\mathrm{FP}},
$$

where

$$
B_{i}^{\mathrm{FP}}=\sum_{j, k}^{j \geqslant k}\left(1-\frac{1}{2} \delta_{j k}\right) \eta(\tilde{v}) \beta_{j k} N_{j} N_{k},
$$

such that $v_{i-1} \leq v_{j}+v_{k} \leq v_{i+1}$ and

$$
D_{i}^{\mathrm{FP}}=D_{i}^{\mathrm{CA}}=N_{i} \sum_{k=1}^{N_{c}} \beta_{i, k} N_{k} .
$$

Here, $\tilde{v}=v_{k}+v_{j}$ and $\beta_{j, k}=\beta\left(t, v_{j}, v_{k}\right)$ is the agglomeration kernel. The expression $\eta(\tilde{v})$ for each particle birth assignment is given by Eq. 34,

$$
\eta(\tilde{v})= \begin{cases}\frac{v_{i+1}-\tilde{v}}{v_{i+1}-v_{i}}, & v_{i} \leq \tilde{v}<v_{i+1} \\ \frac{\tilde{v}-v_{i-1}}{v_{i}-v_{i-1}}, & v_{i-1} \leq \tilde{v}<v_{i} .\end{cases}
$$

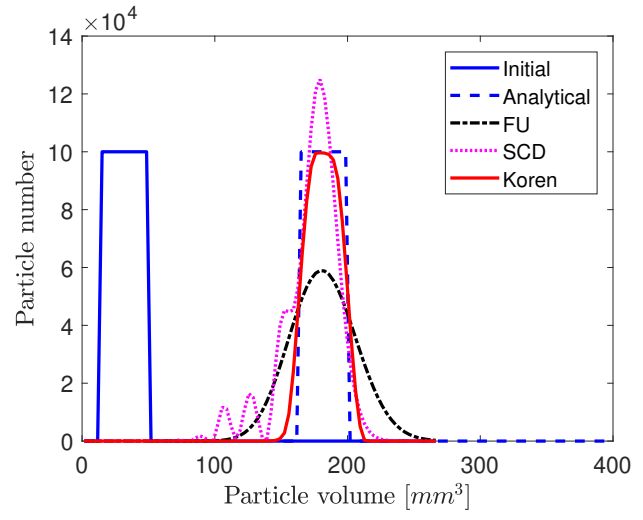

Figure 2. Comparison of PSDs with different numerical schemes for a layering batch process.

\subsection{Kumar et al.'s new finite volume scheme}

Recently, an accurate and efficient discretization method for agglomeration PBE was proposed in (Kumar et al., 2016) based on the finite volume approach. This scheme has an improvement over the finite volume scheme proposed by (Forestier and Mancini, 2012). The scheme provides better solution of several moments in addition to the mass conservation property compared to the scheme in (Forestier and Mancini, 2012). The Kumar et al.'s new finite volume (NFV) scheme assumes the number density function as the point masses concentrated on the cell representatives. The discrete PBE using Kumar et al.'s (Ku-

Table 1. Parameters used to solve PBE for particle layering process.

\begin{tabular}{ll}
\hline Parameter & Layering \\
\hline Range of $v\left[\mathrm{~mm}^{3}\right]$ & $0-400$ \\
Number of cells & 80 \\
Grid type & linear \\
$G\left[\mathrm{~mm}^{3} \cdot \mathrm{s}^{-1}\right]$ & 1 \\
Time step for RK4 $[\mathrm{s}]$ & 0.1 \\
\hline
\end{tabular}

mar et al., 2016) scheme is then given as

$$
\frac{d n_{i}^{\mathrm{NFV}}}{d t}=\frac{1}{2} \sum_{(j, k) \in Q^{i}} \beta_{j, k} n_{j} n_{k} \frac{\Delta v_{j} \Delta v_{k}}{\Delta v_{i}} S_{i, j, k}-\sum_{j=1}^{N_{c}} \beta_{i, j} n_{i} n_{j} \Delta v_{j}
$$

where factor $S_{i, j, k}$ accounts for mass conservation and is defined as

$$
S_{i, j, k}=\frac{v_{j}+v_{k}}{v_{i}} .
$$

The set $Q^{i}$ be a set that contains the pair of cells $j$ and $k$ such that the sum of the cell's representatives, $v_{j}+v_{k}$, falls in the domain of cell $i$ represented by cell node $v_{i}$ :

$$
Q^{i}=\left\{(j, k) \in N_{c} \times N_{c}: v_{i-\frac{1}{2}}<v_{j}+v_{k} \leq v_{i+\frac{1}{2}}\right\} .
$$

In the NFV scheme, to ensure that no mass leaves the 


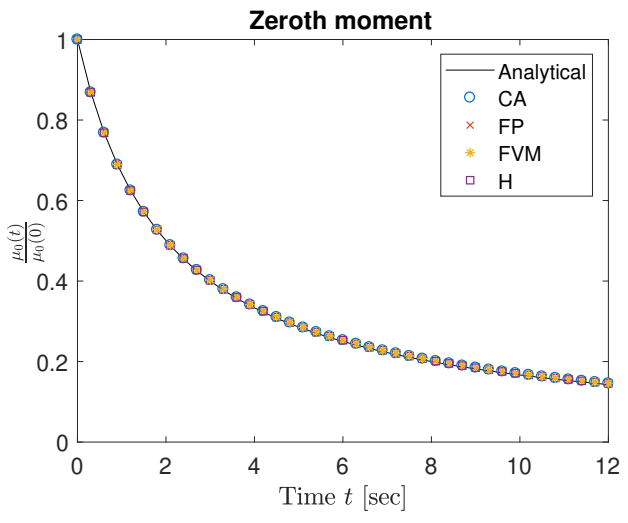

Figure 3. Numerical results for zeroth moment using various numerical schemes for particle agglomeration.

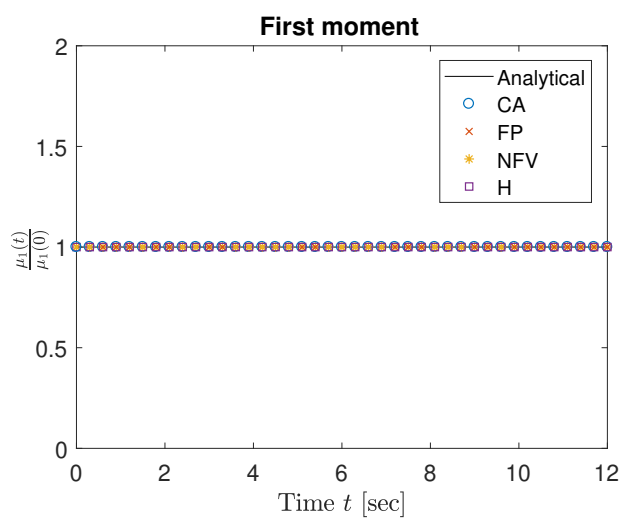

Figure 4. Mass conservation with various numerical schemes.

upper boundary of the size domain (for mass conservation),

$$
\beta\left(v_{j}, v_{k}\right)= \begin{cases}\beta\left(v_{j}, v_{k}\right), & \left(v_{j}+v_{k}\right) \leq v_{\max } \\ 0, & \text { otherwise, }\end{cases}
$$

where $v_{\max }$ is the maximum particle volume.

Table 2. Parameters used to solve PBE for binary particle agglomeration process.

\begin{tabular}{ll}
\hline Parameter & Agglomeration \\
\hline Range of $v\left[\mathrm{~mm}^{3}\right]$ & $0-400$ \\
Number of cells & 15 \\
Grid type & geometric \\
$\beta_{0}\left[\mathrm{~s}^{-1}\right]$ & 1 \\
Time step for RK4 $[\mathrm{s}]$ & 0.1 \\
$N_{0}$ & 1 \\
$v_{0}\left[\mathrm{~mm}^{3}\right]$ & 1 \\
\hline
\end{tabular}

\section{Simulation Results and Discussion}

\subsection{Simulation Setup}

The comparison of different numerical schemes is performed by applying corresponding discretization methods

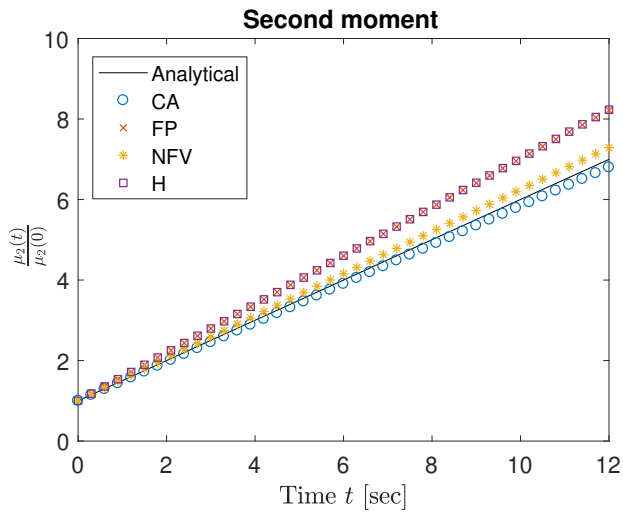

Figure 5. Numerical results for second moment using the various numerical schemes for pure agglomeration.

to batch processes as described in Section 3 and Section 4. Only batch processes with number based PBE with volume as the particle size, are used in these simulations. Such simplified processes are used in order to asses the performance of numerical schemes by comparing their numerical solutions with the analytically tractable solution.

The semi-discrete form (set of ODEs) of the PBEs obtained from particle class size are solved using a 4-th order Runge-Kutta method with fixed time step. Dynamic simulations are performed using MATLAB (MATLAB, 2017). Chosen simulation settings used to asses the accuracy of the numerical schemes for the particle layering process are given in Table 1 while for the particle agglomeration process in Table 2.

\subsection{Comparison of numerical solutions for layering process}

In order to compare the numerical schemes for particle growth due to layering, the number based PBE is utilized as discussed in Section 3. All the simulation cases for the granulation process due to layering are carried out using linear grid for particle size discretization. The entire particle size range is divided into 80 uniformly distributed cells (classes) and a constant growth rate is assumed (see Table 1). The initial PSD distribution is chosen as

$$
N(v, 0)= \begin{cases}10 \times 10^{4} & 15 \leq v \leq 50 \\ 0 & \text { otherwise }\end{cases}
$$

Three finite volume schemes, i.e., first order upwind (FU), second order central difference (SCD), and Koren flux limiter (KFL) schemes are applied to simulate a pure layering batch process. In addition, simulations with an analytically tractable solution (Eq. 5) are carried out to reliably evaluate the accuracy of the numerical schemes. Simulation results (Figure 2) showed that the resulting particle number distribution obtained using the FU scheme has a diffusive behavior but the solution is stable and smooth. Since a significantly smeared solution is observed, the accuracy of the FU scheme is considered to be low. The numerical result obtained with the SCD scheme 
(Figure 2) should have a higher accuracy compared to the FU scheme owing to its higher order. This, however, is true only for smooth solutions. The SCD scheme produces oscillations when the solution changes abruptly, i.e., in the presence of discontinuous analytical solutions. Fortunately, the oscillations that appear in the solution with the SCD scheme do not appear in the numerical solution when the KFL scheme was used. Thus, the highest accuracy among the three tested finite volume schemes is achieved using the finite volume scheme extended with the Koren flux limiter, Figure 2. Higher accuracy of the latter scheme is achieved since in the smooth solution regions, the Koren flux limiter function (in general a second order flux limiter) shows second order accuracy, while in the region where discontinuities appear, the flux limiter acts like a first order scheme, and thus produces a smooth solution. The drawback of the solution obtained with the KFL scheme is the increased computational time: the FU and SCD schemes can be solved relatively faster than the KFL scheme.

\subsection{Comparison of numerical solutions for ag- glomeration process}

Comparison of numerical schemes for the pure agglomeration process is carried out on a geometric grid with a factor of 2 in size $\left(v_{i+1}=2 v_{i}\right)$ using 15 cells (Table 2$)$.

The four different numerical schemes that are applied to the process for comparison are: Hounslow $(\mathrm{H})$, fixed pivot (FP), cell average (CA), and a new Kumar et al.'s (2016) finite volume (NFV) scheme. The moments are calculated using discrete form of Eq. 2:

$$
\mu^{l}=\sum_{i}^{N c} x^{l} \Delta x_{i} n_{i}
$$

Assessment of the accuracy of the numerical schemes is performed by comparing the numerical solutions with an analytically tractable solution as mentioned in Section 4.

Simulation results showed that three tested numerical schemes produce solutions where conservation of the zeroth moment (total number conservation) and the first moment (mass conservation) are fulfilled (Figure 3 and Figure 4). No deviation is observed between the analytical solution and the numerical solutions for the first two moments for any of the numerical schemes.

However, the discrepancy between the numerical solutions and the analytical solution shows up for higher moments (second moment, as shown in Figure 5). The best accuracy for the second moment is observed for the numerical solution using the CA scheme.

The NFV scheme also predicts the second moment sufficiently accurate. The poorest solution accuracy for the second moment is produced when the $\mathrm{H}$ and the FP schemes are used. In addition, these two sectional schemes produce the same simulation results. This is due to the specific choice of the grid for particle size discretization: The FP scheme becomes equivalent to the $\mathrm{H}$

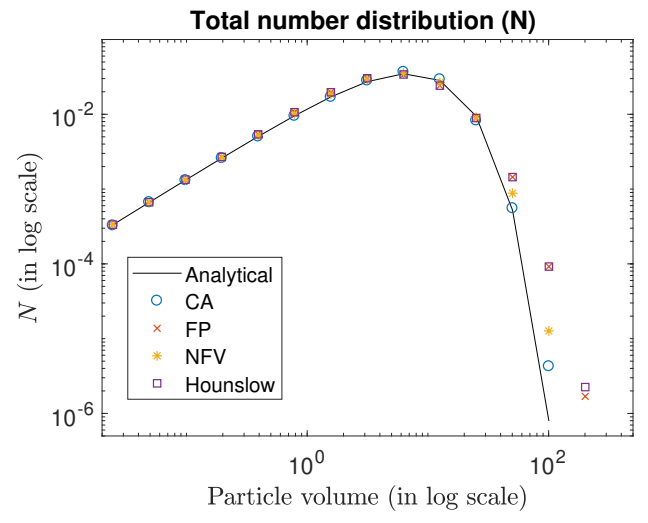

Figure 6. Simulation results for the total number distribution.

scheme when the geometric grid is used with a factor of 2 (Kumar, 2006). Thus, the FP and H schemes should produce the same results when such geometric discretization is used.

Another significant task is to evaluate the performance of the numerical schemes to predict the particle number distribution, usually visualized in log scale as shown in Figure 6. As the particles flow towards the higher size range in the agglomeration process, it is interesting to see how the numerical schemes perform at higher particle size range (thus log scale is used for improved visualization). Here, again, we can see the the discrepancy between the numerical and the analytical solutions. For lower particle size range, all the numerical schemes predicted accurately the particle number distribution. However, for higher particle size range, deviations from the analytical solution are observed. Among the tested numerical schemes, the FP and $\mathrm{H}$ schemes over-predict the actual results and thus show the poorest prediction of the particle number distribution. Similar to the moments prediction, the CA scheme and the NFV scheme show relatively better agreement with the analytical solution even for a coarse grid ( 15 cells).

\section{Conclusions}

Comparison of numerical schemes for solving population balance equations is presented in this paper, for pure layering and pure agglomeration problems. Discretization of the layering term is performed by applying various finite volume schemes. Among the three tested approximation schemes, the Koren flux limiter scheme exhibits relatively better performance in terms of accuracy. However, the Koren scheme also needs a higher computational time compared to the other tested numerical schemes. Numerical solutions for the agglomeration process were obtained by applying different sectional methods, as well as a recent finite volume scheme. The numerical performance of the cell average scheme and the new finite volume scheme in predicting the particle size distribution at higher particle size range is relatively better than Hounslow's scheme and the fixed pivot scheme. The former schemes produce a 
PSD that is in good agreement with the analytical solution with coarser grid.

\section{Acknowledgment}

The economic support from The Research Council of Norway and Yara Technology Centre through project no. 269507/O20 'Exploiting multi-scale simulation and control in developing next generation high efficiency fertilizer technologies (HEFTY)' is gratefully acknowledged.

\section{References}

L. Forestier and S. Mancini. A finite volume preserving scheme on nonuniform meshes and for multidimensional coalescence. SIAM Journal of Scientific Computing, 34(6), 2012. doi:10.1137/110847998.

S. Heinrich, M. Peglow, M. Ihlow, and L. Mörl. Particle population modeling in fluidized bed-spary granulaiton - analysis of the steady state and unsteady behavior. Powder Technology, 130:154-161, 2003. doi:10.1016/S0032-5910(02)00259-0.

MJ Hounslow, RL Ryall, and VR Marshall. A discretized population balance for nucleation, growth, and aggregation. AIChE Journal, 34(11):1821-1832, 1988.

H.M. Hulburt and S. Katz. Some problems in particle technology: A statistical mechanical formulation. Chemical Engineering Science, 19(8):555-574, 1964.

G Kaur, J. Kumar, and S. Heinrich. A weighted finite volume scheme for multivariate aggregation population balance equation. Computers \& Chemical Engineering, 101:1-10, 2017.

B. Koren. A robust upwind discretization method for advection, diffusion and source terms. In C. B. Vreugdenhil and B. Koren, editors, Numerical Methods for AdvectionDiffusion Problems, Notes on Numerical Fluid Mechanics, pages 117-138. 1993.

J. Kumar. Numerical approximations of population balance equations in particulate systems. $\mathrm{PhD}$ thesis, Otto-von-Guericke-Universität Magdeburg, Universitätsbibliothek, 2006.

J. Kumar, M. Peglow, G. Warnecke, S. Heinrich, and L. Mörl. Improved accuracy and convergence of discretized population balance for aggregation: The cell average technique. Chemical Engineering Science, 61(10):3327-3342, 2006.

J. Kumar, G. Kaur, and E. Tsotsas. An accurate and efficient discrete formulation of aggregation population balance equation. Kinetic \& Related Models, 9(2), 2016.

S. Kumar and D. Ramkrishna. On the solution of population balance equations by discretization - i. a fixed pivot technique. Chemical Engineering Science, 51(8):1311-1332, 1996.

J. Litster and B. Ennis. The science and engineering of granulation processes, volume 15. Springer Science \& Business Media, 2004.

MATLAB. 2017a. The MathWorks, Inc., Natick, Massachusetts, United States., 2017.
R. Radichkov, T. Müller, A. Kienle, S. Heinrich, M. Peglow, and L. Mörl. A numerical bifurcation analysis of continuous fluidized bed spray granulator with external product classification. Chemical Engineering and Processing, 45:826-837, 2006. doi:10.1016/j.cep.2006.02.003.

D. Ramkrishna. Population balances: Theory and applications to particulate systems in engineering. Academic press, 2000.

WT Scott. Analytic studies of cloud droplet coalescence i. Journal of the atmospheric sciences, 25(1):54-65, 1968.

M. Singh, J. Kumar, and A. Bück. A volume conserving discrete formulation of aggregation population balance equations on non-uniform meshes. IFAC-PapersOnLine, 48(1):192-197, 2015. 